\title{
$4 f-\pi$ Molecular Hybrid Exhibiting Rich Conductive Phases and Slow Relaxation of Magnetization
}

Yongbing Shen, ${ }^{1 *}$ Goulven Cosquer, ${ }^{2}$ Haitao Zhang, ${ }^{3}$ Brian K. Breedlove, ${ }^{1}$ Mengxing Cui, ${ }^{1}$ and Masahiro Yamashita, ${ }^{1,4, *}$

${ }^{1}$ Department of Chemistry, Graduate School of Science, Tohoku University, 6-3 Aza-Aoba, Aramaki, Aoba-ku, Sendai 980-8578, Japan.

${ }^{2}$ Research Group of Solid Material Chemistry, Graduate School of Science, Hiroshima University, 1-31 Kagamiyama, Higashihiroshima, Hiroshima 739-8526, Japan

${ }^{3}$ Institute of Inorganic and Applied Chemistry, University of Hamburg, Martin-Luther-King-Platz 6, 20146 Hamburg, Germany

${ }^{4}$ School of Materials Science and Engineering, Nankai University, Tianjin 300350, China. 


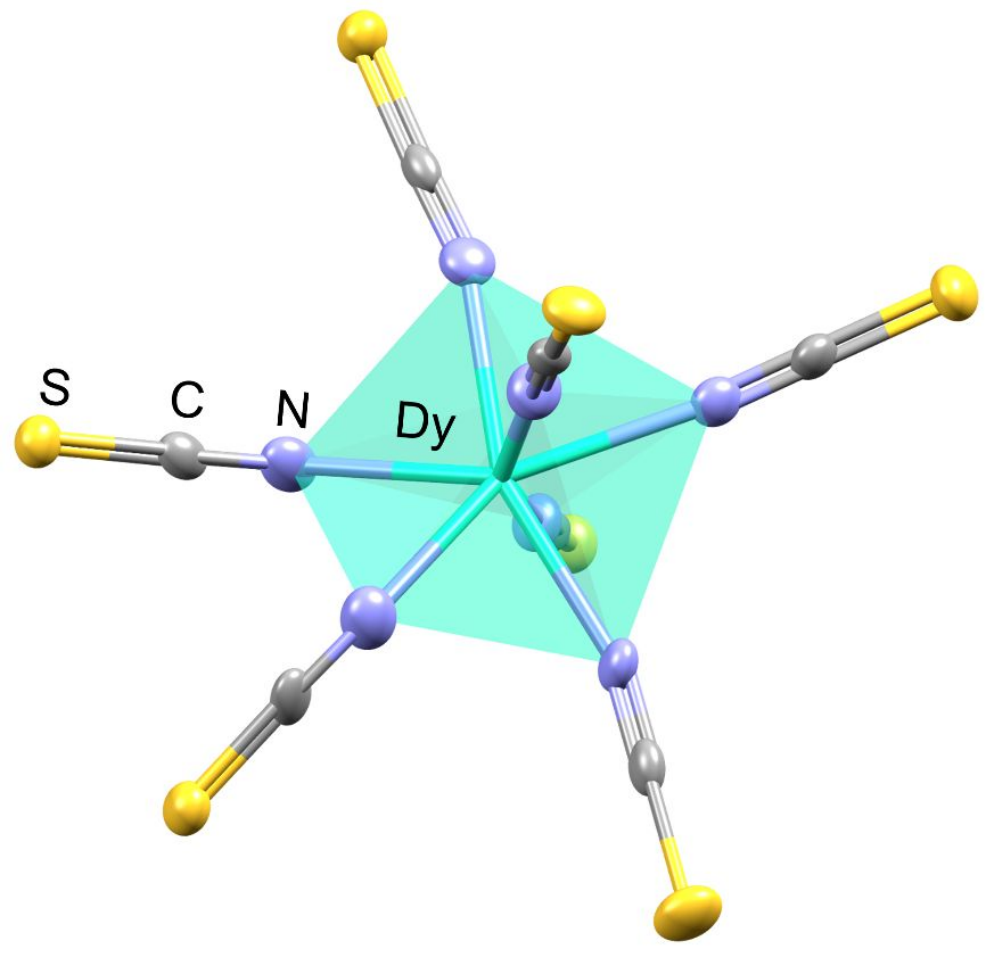

Figure S1. The coordination geometry of $\left[\mathrm{Dy}(\mathrm{NSC})_{7}\right]^{4-}$ complex. 


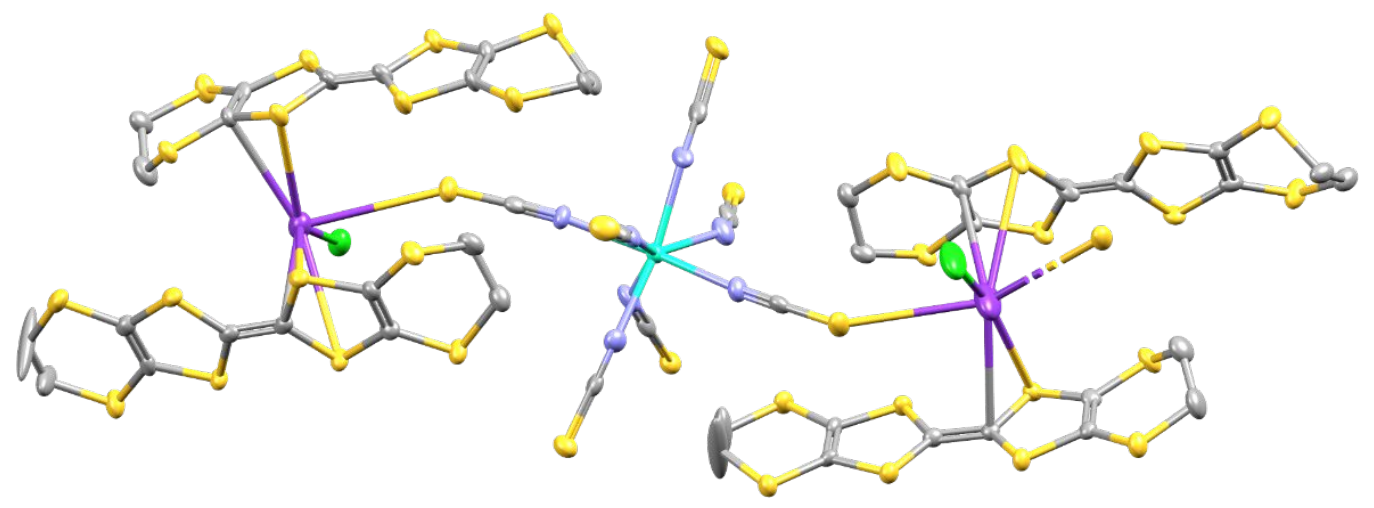

Figure S2. The localized structure of $\mathbf{1}$. The $\mathrm{K}^{+}$ions of $\mathrm{KCl}$ salts have thermal ellipsoids at $25 \%$ probability. Each $\mathrm{K}^{+}$ion coordinated with two $\mathrm{ET}^{+\bullet}$ radicals, which were interlinked by $\left[\mathrm{Dy}(\mathrm{NCS})_{7}\right]^{4-}$ complex through $\mathrm{NCS}^{-}$ligand. 


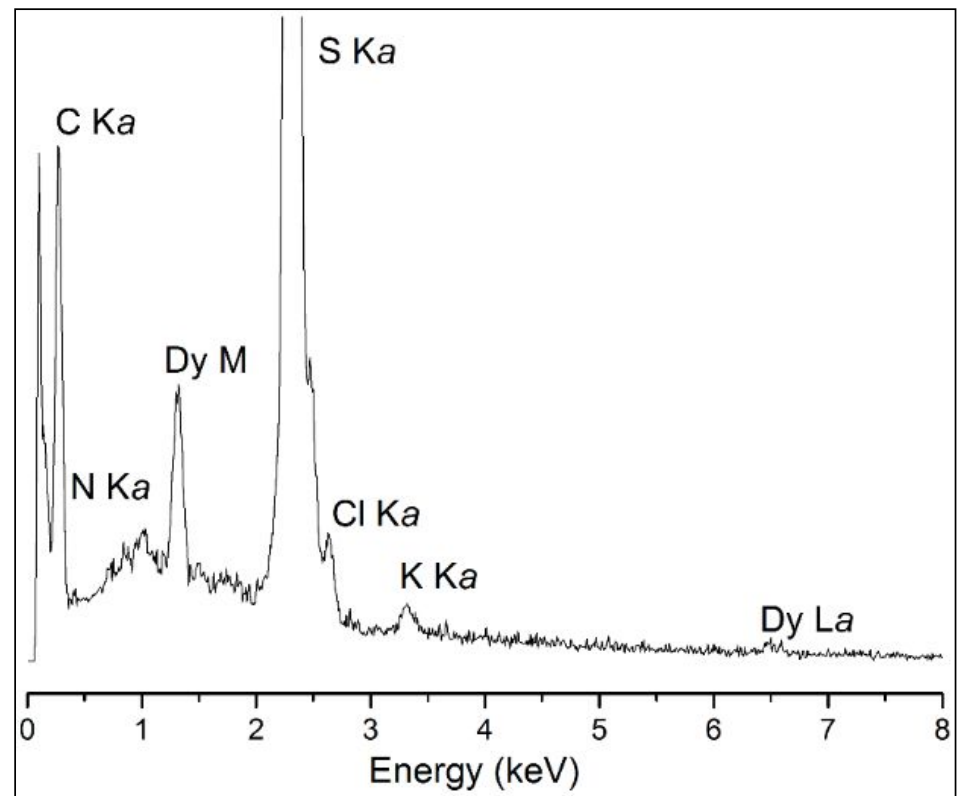

Chemical formula $\quad \mathrm{C}_{57} \mathrm{H}_{40} \mathrm{~N}_{7} \mathrm{~S}_{57} \mathrm{Dy}_{1} \mathrm{~K}_{0.5} \mathrm{Cl}_{0.5}$

\begin{tabular}{cccccc}
\hline Elements & $\mathrm{C}$ & $\mathrm{H}$ & $\mathrm{N}$ & $\mathrm{S}$ & $\mathrm{Cl}$ \\
\hline Calcd Value (\%) & 24.02 & 1.41 & 3.44 & 64.12 & 0.62 \\
& & & & & \\
\hline Found Value (\%) & 23.98 & 1.88 & 3.53 & 64.21 & 0.78 \\
\hline
\end{tabular}

Figure S3. Energy-dispersive X-ray (EDX) spectrum and elemental analysis. 


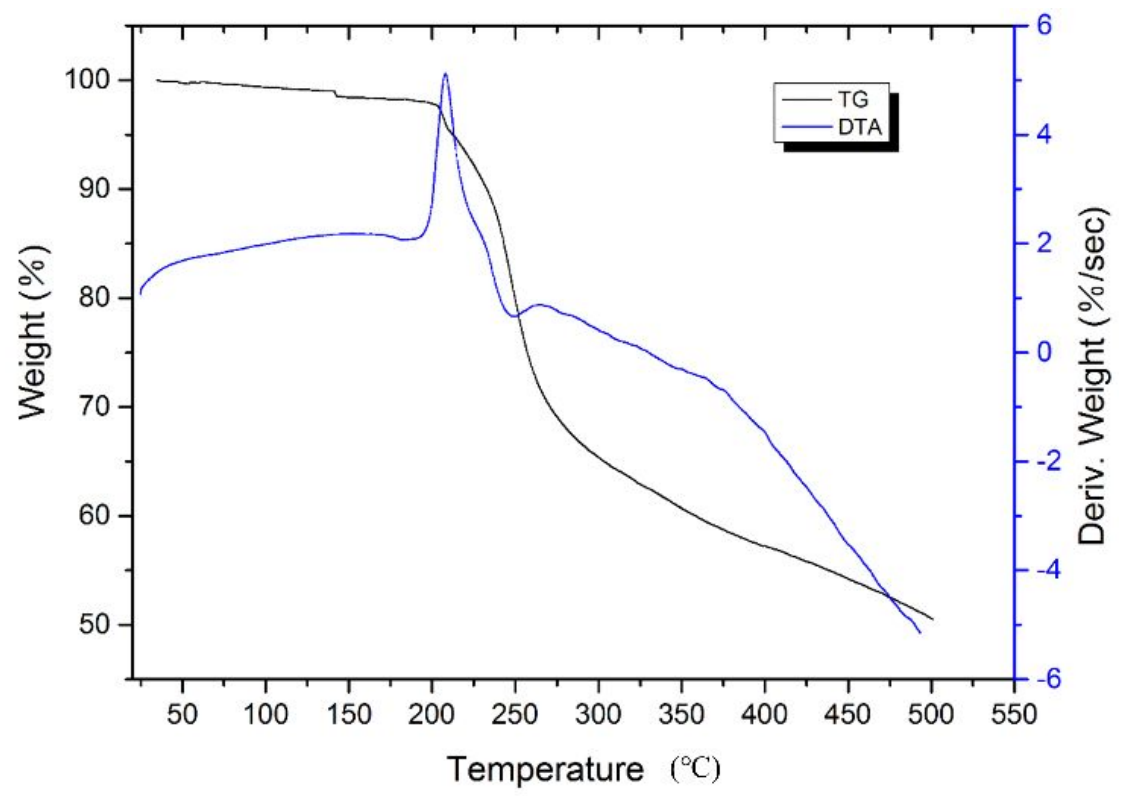

Figure S4. Thermogravimetric analysis (TGA). 


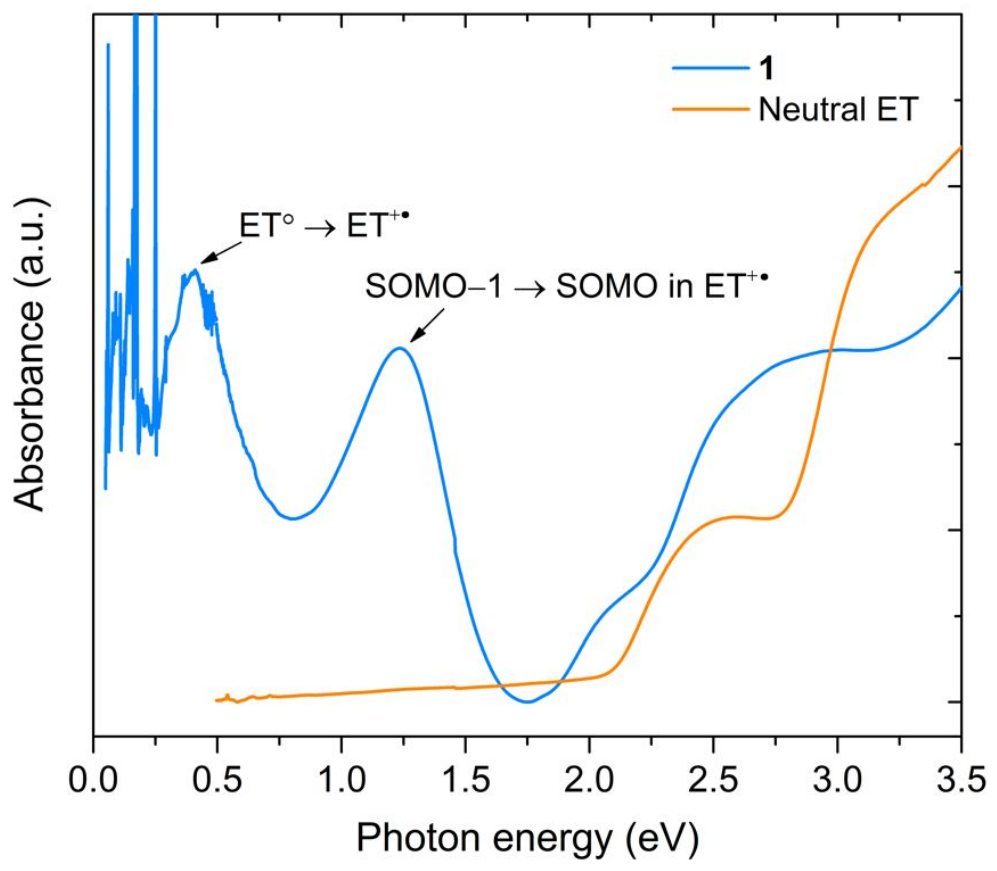

Figure S5. Spectroscopic properties. Room-temperature solid-state absorbance spectra in the range of $0.05-3.5 \mathrm{eV}$. 


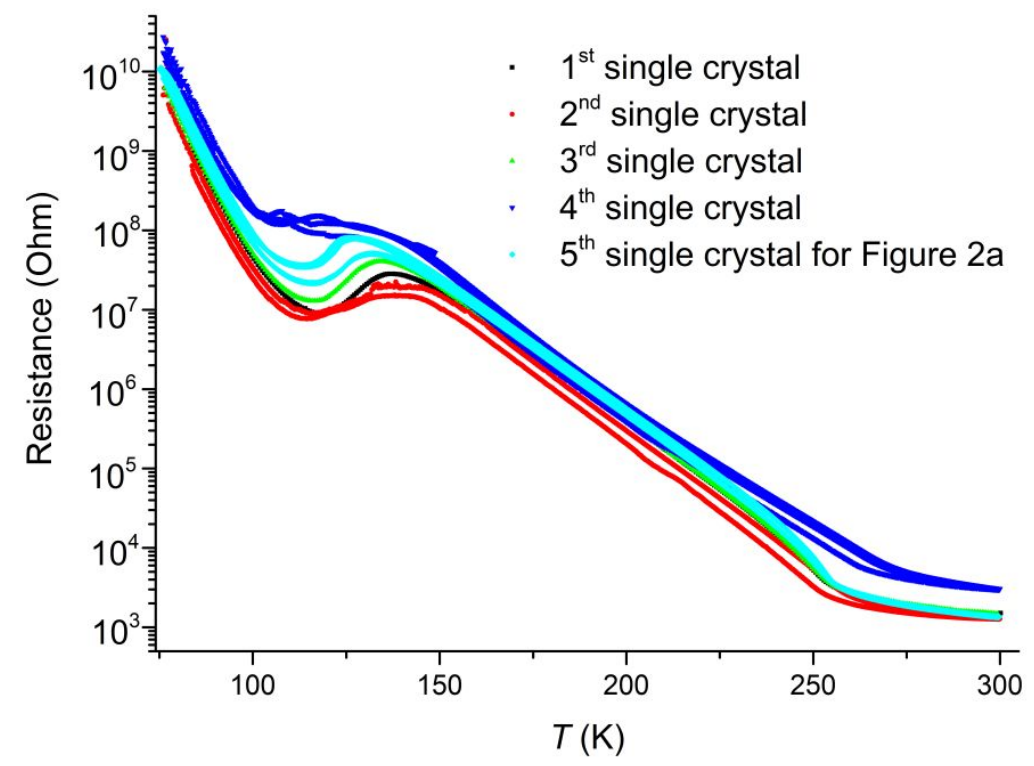

Figure S6. Temperature-dependence of electrical conductivity by using several single-crystals. 


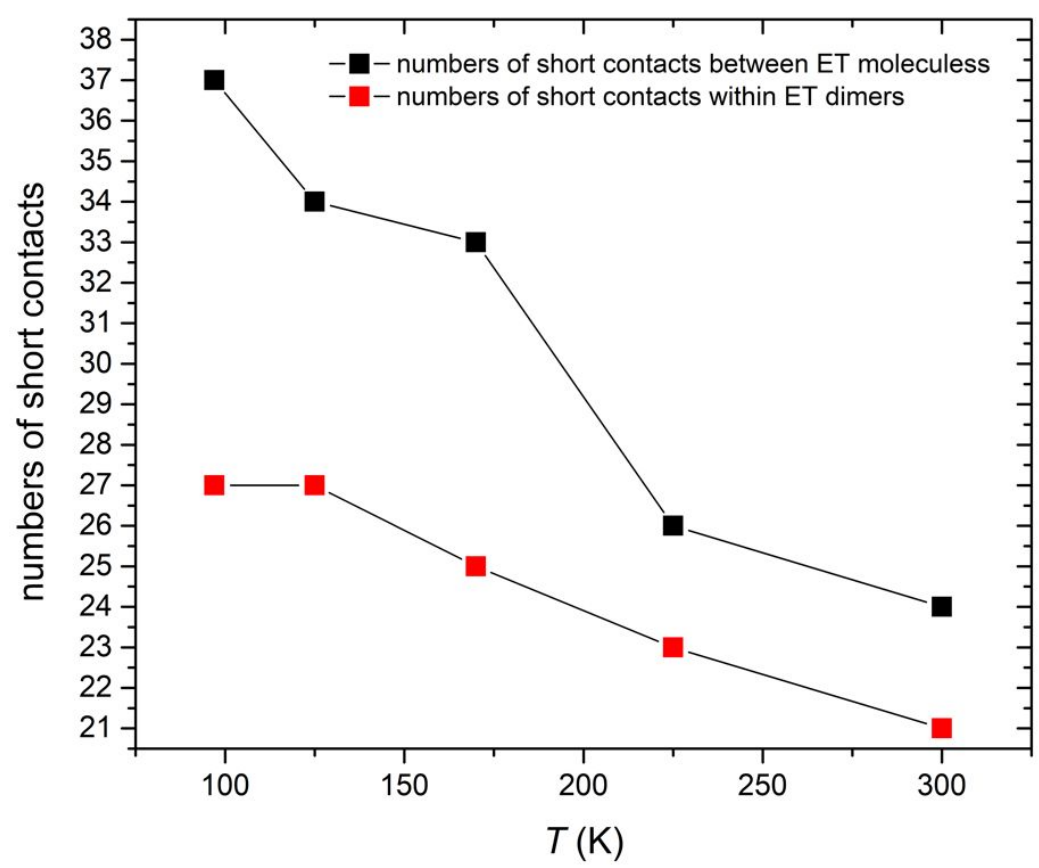

Figure S7. Numbers of short contacts between ET molecules and within ET dimers at various temperature points. 


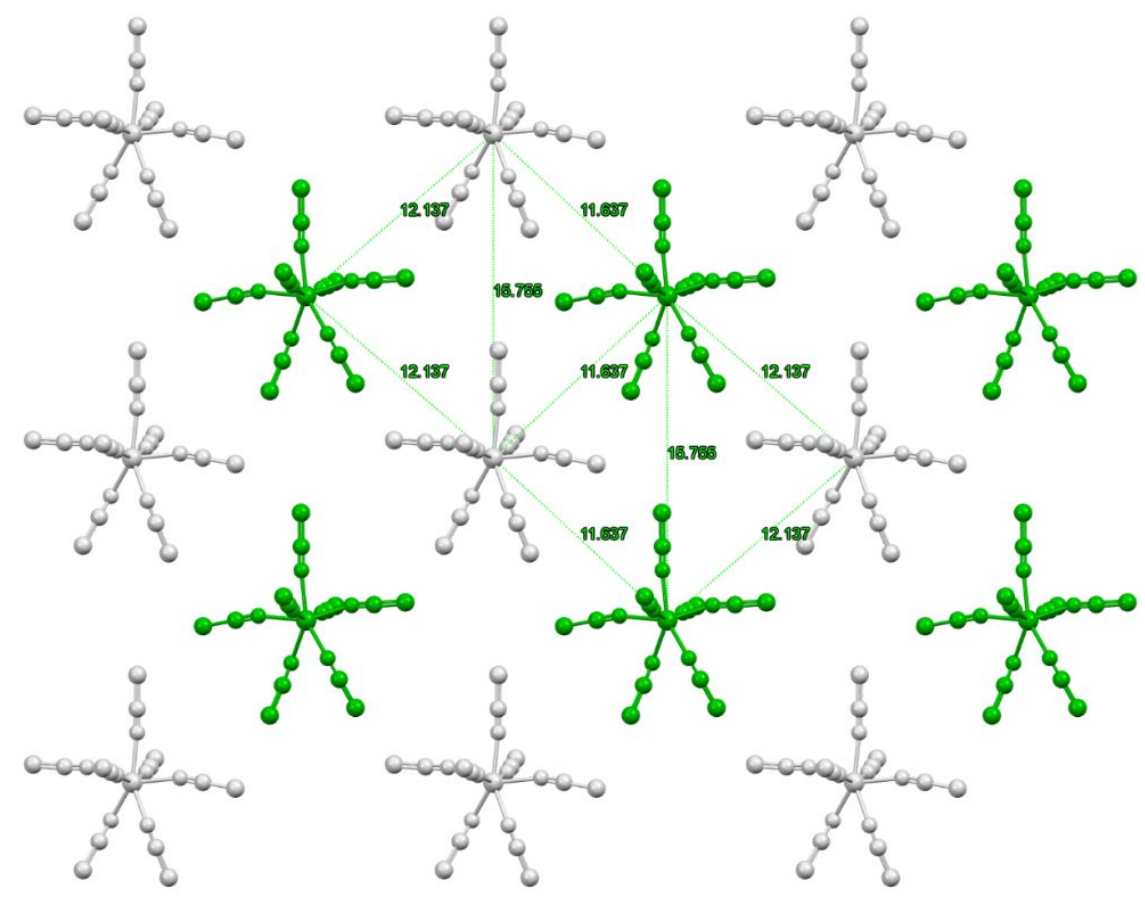

Figure S8. The packing structure of $\left[\mathrm{Dy}(\mathrm{NSC})_{7}\right]^{4-}$ complexes in magnetic layer. 


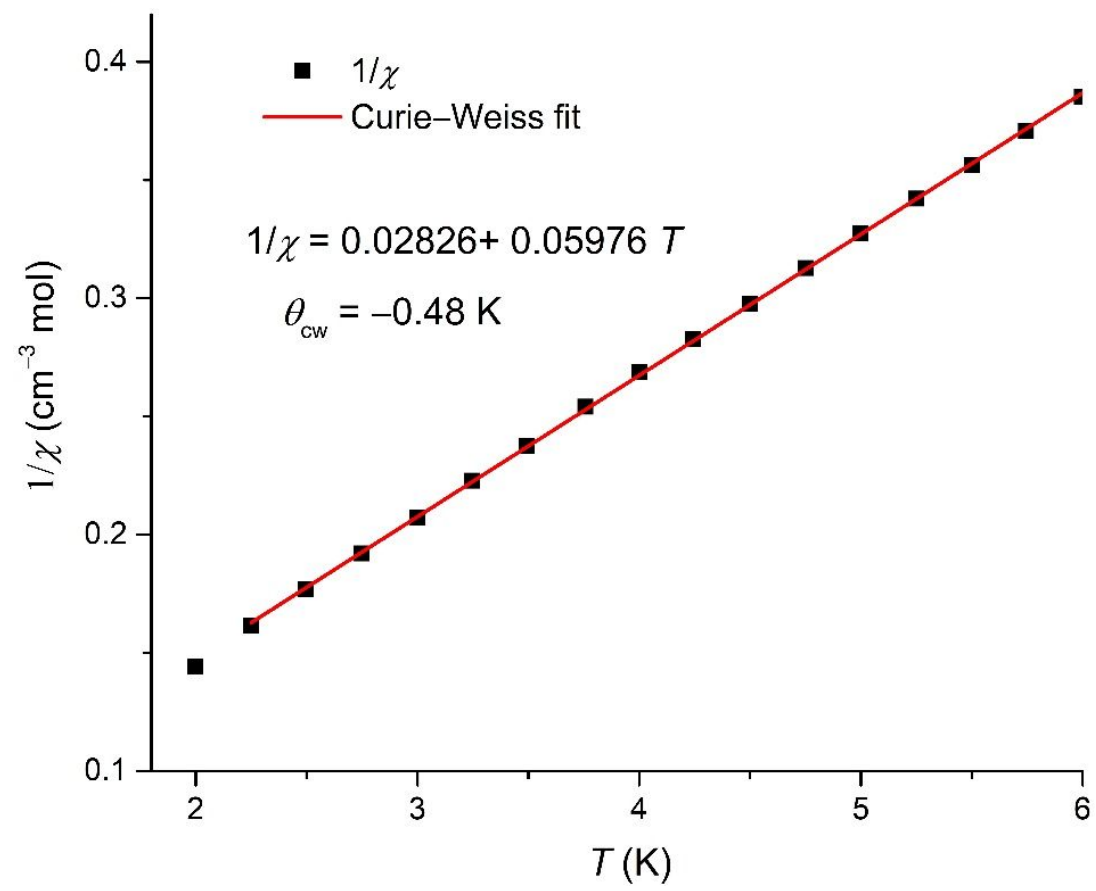

Figure S9. $1 / \chi-T$ plot of 1 in a temperature range of $2-6 \mathrm{~K}$. The red line represents the best fit by using the Curie-Weiss law. 


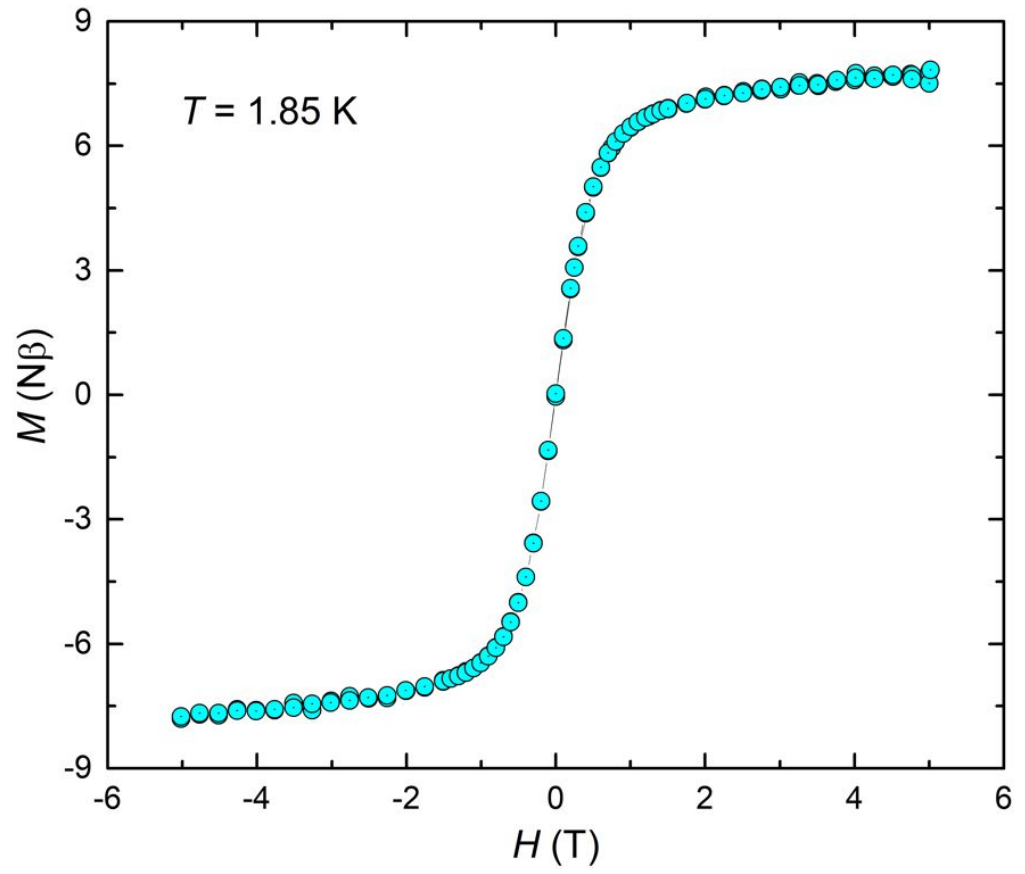

Figure S10. The magnetic field dependence of magnetization $(\mathrm{MH})$ at $1.85 \mathrm{~K}$. 


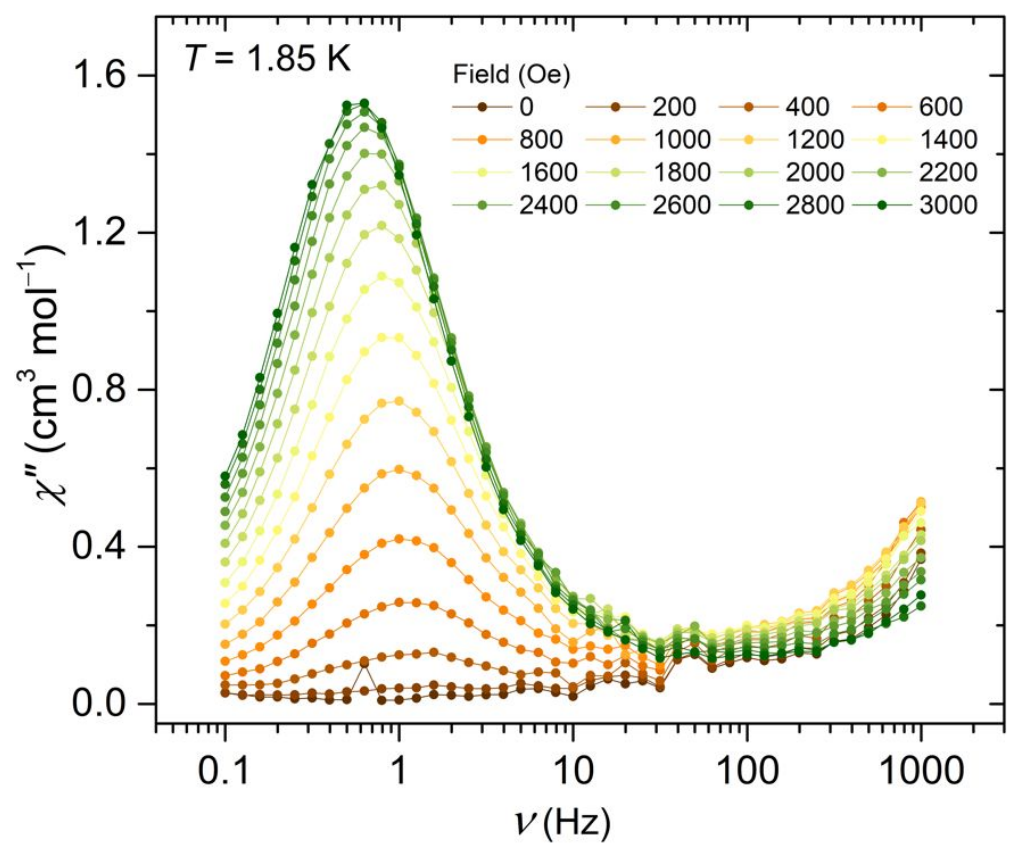

Figure S11. Frequency-dependence of $\chi^{\prime \prime}$ in the field range of $0-3000$ Oe at $1.85 \mathrm{~K}$. 


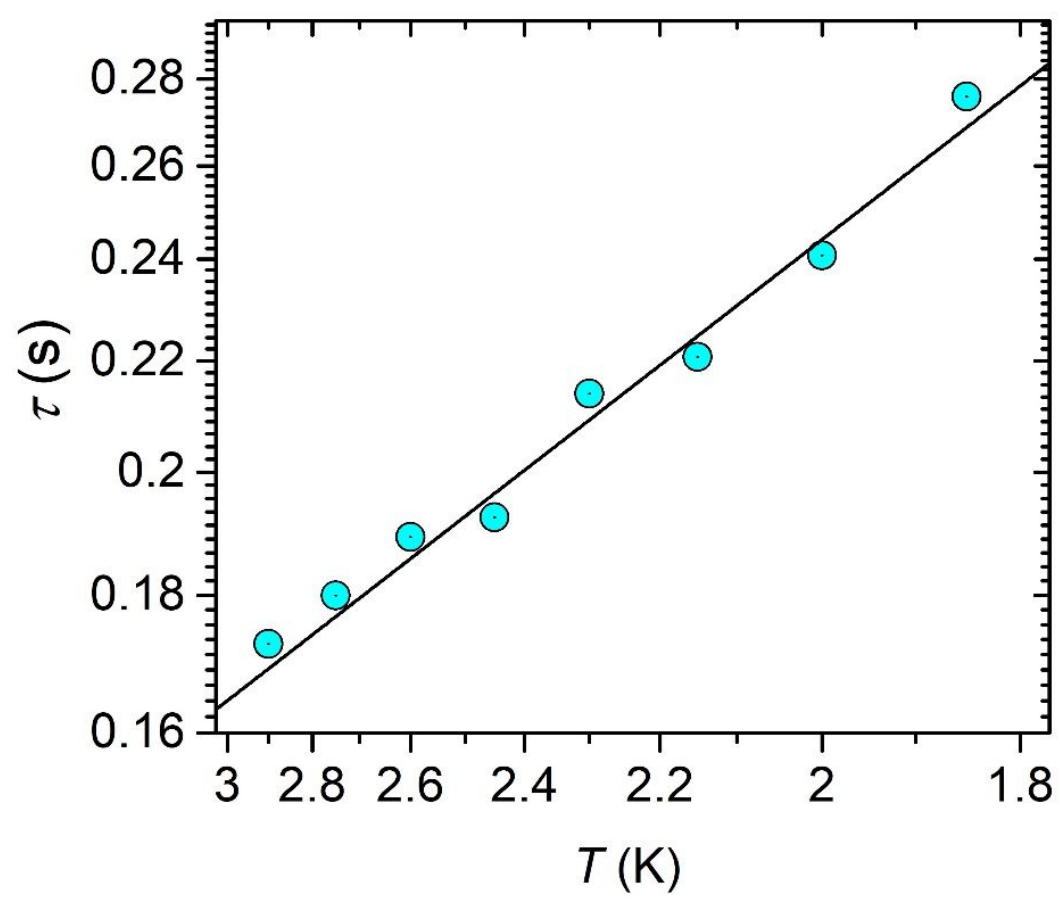

Figure S12. Temperature-dependence of magnetic relaxation time $(\tau)$ in applied 3000 Oe field obtained from the first relaxation process. The black line represents the best fit by using direct process. 
Table S1. The crystallographic data of $\mathbf{1}$ at various temperatures.

\begin{tabular}{|c|c|c|c|c|c|}
\hline & $97 \mathrm{~K}$ & $125 \mathrm{~K}$ & $170 \mathrm{~K}$ & $225 \mathrm{~K}$ & $300 \mathrm{~K}$ \\
\hline Formula & \multicolumn{5}{|c|}{$\mathrm{Dy}_{1} \mathrm{~N}_{7} \mathrm{C}_{57} \mathrm{~S}_{57} \mathrm{H}_{40} \mathrm{~K}_{0.5} \mathrm{Cl}_{0.5}$} \\
\hline Crystal system & \multicolumn{5}{|c|}{ Monoclinic } \\
\hline Space group & \multicolumn{5}{|c|}{$P 2_{1} / \mathrm{n}$} \\
\hline$a(\AA)$ & $17.734(2)$ & $17.83(2)$ & $17.880(8)$ & $17.987(7)$ & $18.0922(11)$ \\
\hline$b(\AA)$ & $15.713(2)$ & $15.814(17)$ & $15.834(7)$ & $15.879(6)$ & $15.9213(9)$ \\
\hline$c(\AA)$ & $32.477(4)$ & $32.70(4)$ & $32.645(14)$ & $32.717(12)$ & $32.810(2)$ \\
\hline$\beta\left({ }^{\circ}\right)$ & $100.059(5)$ & $100.059(7)$ & $99.976(5)$ & $99.841(4)$ & $99.493(3)$ \\
\hline$V\left(\AA^{3}\right)$ & $8910.7(19)$ & $9078(18)$ & $9102(7)$ & $9207(6)$ & $9321.5(10)$ \\
\hline \multirow[t]{3}{*}{$\begin{array}{l}\text { Index } \\
\text { range }\end{array}$} & $-17<\mathrm{h}<9$ & $-18<\mathrm{h}<18$ & $-18<\mathrm{h}<12$ & $-18<\mathrm{h}<12$ & $-18<\mathrm{h}<18$ \\
\hline & $-16<\mathrm{k}<11$ & $-15<\mathrm{k}<7$ & $-12<\mathrm{k}<16$ & $-12<\mathrm{k}<16$ & $-16<\mathrm{k}<9$ \\
\hline & $-34<1<34$ & $-34<1<18$ & $-27<1<30$ & $-27<1<30$ & $-34<1<21$ \\
\hline$F(000)$ & 5672 & 5880 & 5672 & 5744 & 5840 \\
\hline Radiation & \multicolumn{5}{|c|}{ MoK $\backslash \mathrm{a} 0.71$} \\
\hline$\theta_{\min }$ & 4.037, & 4.010 & 4.015, & 4.004, & 4.026 , \\
\hline$\theta_{\max }\left({ }^{\circ}\right)$ & 22.335 & 22.147 & 22.037 & 22.166 & 22.130 \\
\hline GOF on $\mathrm{F}^{2}$ & 1.071 & 1.004 & 0.979 & 0.982 & 0.871 \\
\hline$R_{1} / \mathrm{w} R_{2}(\mathrm{gt})$ & $0.0590 / 0.1752$ & $0.0639 / 0.1796$ & $0.0596 / 0.1668$ & $0.0595 / 0.1645$ & $0.0597 / 0.1586$ \\
\hline
\end{tabular}


Table S2. The bond lengths of each ET molecule and the calculated charges at various temperatures.

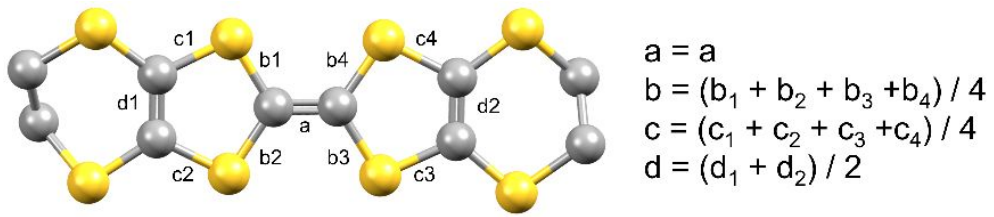

\begin{tabular}{|c|c|c|c|c|c|c|c|c|c|c|c|c|c|c|c|c|}
\hline$T / \mathrm{K}$ & ET & $\mathbf{a} / \boldsymbol{\AA}$ & b1 $1 / \boldsymbol{A}$ & $\mathbf{b} 2 / \boldsymbol{\AA}$ & $\mathbf{b} \mathbf{3} / \mathbf{A}$ & b4/ $/ \AA$ & c1 $1 / \AA$ & c $2 / \AA$ & c $3 / \AA$ & c4/ $/ \AA$ & $\mathbf{d} \mathbf{1} / \boldsymbol{\AA}$ & $\mathrm{d} 2 / \AA$ & $\mathbf{b} / \mathbf{A}$ & $\mathbf{c} / \boldsymbol{\AA}$ & $\mathrm{d} / \boldsymbol{\AA}$ & $\xi$ \\
\hline \multirow{5}{*}{97} & 1 & 1.387 & 1.735 & 1.698 & 1.716 & 1.726 & 1.728 & 1.734 & 1.728 & 1.757 & 1.32 & 1.376 & 1.71875 & 1.73675 & 1.348 & 0.96991 \\
\hline & 2 & 1.403 & 1.706 & 1.709 & 1.718 & 1.72 & 1.742 & 1.743 & 1.731 & 1.738 & 1.356 & 1.337 & 1.71325 & 1.7385 & 1.3465 & 1.10611 \\
\hline & 3 & 1.381 & 1.726 & 1.703 & 1.757 & 1.721 & 1.745 & 1.746 & 1.734 & 1.751 & 1.341 & 1.365 & 1.72675 & 1.744 & 1.353 & 0.84863 \\
\hline & 4 & 1.377 & 1.707 & 1.713 & 1.735 & 1.729 & 1.746 & 1.751 & 1.744 & 1.729 & 1.355 & 1.35 & 1.721 & 1.7425 & 1.3525 & 0.86916 \\
\hline & 5 & 1.325 & 1.738 & 1.738 & 1.772 & 1.772 & 1.745 & 1.745 & 1.766 & 1.766 & 1.34 & 1.34 & 1.755 & 1.7555 & 1.34 & 0.03703 \\
\hline \multirow{5}{*}{125} & 1 & 1.373 & 1.719 & 1.751 & 1.739 & 1.72 & 1.751 & 1.754 & 1.752 & 1.766 & 1.341 & 1.375 & 1.73225 & 1.75575 & 1.358 & 0.69751 \\
\hline & 2 & 1.359 & 1.737 & 1.733 & 1.736 & 1.729 & 1.765 & 1.752 & 1.761 & 1.767 & 1.364 & 1.355 & 1.73375 & 1.76125 & 1.3595 & 0.55198 \\
\hline & 3 & 1.405 & 1.713 & 1.727 & 1.724 & 1.727 & 1.765 & 1.723 & 1.725 & 1.746 & 1.351 & 1.382 & 1.72275 & 1.73975 & 1.3665 & 1.19007 \\
\hline & 4 & 1.358 & 1.732 & 1.723 & 1.726 & 1.762 & 1.771 & 1.764 & 1.758 & 1.747 & 1.381 & 1.369 & 1.73575 & 1.76 & 1.375 & 0.6546 \\
\hline & 5 & 1.337 & 1.775 & 1.775 & 1.745 & 1.745 & 1.756 & 1.756 & 1.758 & 1.758 & 1.368 & 1.368 & 1.76 & 1.757 & 1.368 & 0.28704 \\
\hline \multirow{5}{*}{170} & 1 & 1.427 & 1.701 & 1.724 & 1.719 & 1.711 & 1.74 & 1.755 & 1.755 & 1.738 & 1.36 & 1.381 & 1.71375 & 1.747 & 1.3705 & 1.39717 \\
\hline & 2 & 1.362 & 1.731 & 1.737 & 1.724 & 1.729 & 1.741 & 1.756 & 1.758 & 1.751 & 1.34 & 1.378 & 1.73025 & 1.7515 & 1.359 & 0.66952 \\
\hline & 3 & 1.376 & 1.72 & 1.72 & 1.735 & 1.726 & 1.773 & 1.752 & 1.753 & 1.766 & 1.355 & 1.377 & 1.72525 & 1.761 & 1.366 & 0.79266 \\
\hline & 4 & 1.395 & 1.715 & 1.725 & 1.733 & 1.723 & 1.745 & 1.731 & 1.75 & 1.75 & 1.381 & 1.333 & 1.724 & 1.744 & 1.357 & 1.00349 \\
\hline & 5 & 1.332 & 1.737 & 1.772 & 1.737 & 1.772 & 1.769 & 1.769 & 1.758 & 1.758 & 1.352 & 1.352 & 1.7545 & 1.7635 & 1.352 & 0.12286 \\
\hline \multirow{5}{*}{225} & 1 & 1.405 & 1.711 & 1.717 & 1.732 & 1.719 & 1.737 & 1.748 & 1.748 & 1.742 & 1.392 & 1.347 & 1.71975 & 1.74375 & 1.3695 & 1.20499 \\
\hline & 2 & 1.36 & 1.727 & 1.732 & 1.722 & 1.735 & 1.743 & 1.743 & 1.765 & 1.736 & 1.351 & 1.367 & 1.729 & 1.74675 & 1.359 & 0.69937 \\
\hline & 3 & 1.402 & 1.73 & 1.7 & 1.731 & 1.718 & 1.752 & 1.769 & 1.76 & 1.728 & 1.36 & 1.38 & 1.71975 & 1.75225 & 1.37 & 1.1229 \\
\hline & 4 & 1.391 & 1.719 & 1.708 & 1.727 & 1.737 & 1.74 & 1.743 & 1.73 & 1.72 & 1.386 & 1.345 & 1.72275 & 1.73325 & 1.3655 & 1.12663 \\
\hline & 5 & 1.346 & 1.745 & 1.745 & 1.756 & 1.756 & 1.773 & 1.773 & 1.752 & 1.752 & 1.344 & 1.344 & 1.7505 & 1.7625 & 1.344 & 0.20495 \\
\hline \multirow{5}{*}{300} & 1 & 1.415 & 1.709 & 1.723 & 1.71 & 1.723 & 1.757 & 1.735 & 1.747 & 1.745 & 1.358 & 1.357 & 1.71625 & 1.746 & 1.3575 & 1.1994 \\
\hline & 2 & 1.397 & 1.734 & 1.722 & 1.701 & 1.714 & 1.745 & 1.748 & 1.747 & 1.743 & 1.366 & 1.339 & 1.71775 & 1.74575 & 1.3525 & 1.01842 \\
\hline & 3 & 1.397 & 1.71 & 1.728 & 1.741 & 1.704 & 1.744 & 1.765 & 1.723 & 1.758 & 1.366 & 1.367 & 1.72075 & 1.7475 & 1.3665 & 1.08745 \\
\hline & 4 & 1.393 & 1.726 & 1.71 & 1.714 & 1.736 & 1.744 & 1.738 & 1.717 & 1.728 & 1.381 & 1.337 & 1.7215 & 1.73175 & 1.359 & 1.11357 \\
\hline & 5 & 1.34 & 1.756 & 1.756 & 1.748 & 1.748 & 1.76 & 1.76 & 1.767 & 1.767 & 1.33 & 1.33 & 1.752 & 1.7635 & 1.33 & 0.03703 \\
\hline
\end{tabular}

